\title{
A Virtual Assistant for Web-Based Training In Engineering Education
}

\author{
Frédéric Geoffroy $^{(1)}$, Esma Aimeur ${ }^{(2)}$, and Denis Gillet ${ }^{(1)}$ \\ ${ }^{(1)}$ Swiss Federal Institute of Technology in Lausanne (EPFL) \\ LA-I2S-STI, CH - 1015 Lausanne, Switzerland \\ Phone: +41 21 693-5168, FAX: + 4121 693-2574, E-mail: d, \\ frederic.geoffroy@epfl.ch, denis.gillet@epfl.ch \\ ${ }^{(2)}$ Université de Montréal, \\ Département IRO \\ C.P. 6128, Succursale Centre-Ville, Montréal (Québec), H3C 3J7 Canada \\ aimeur@IRO. UMontreal.CA
}

\begin{abstract}
Experimentation has always been an essential ingredient to sustain the learning activities in engineering education. During traditional laboratory sessions, a huge amount of work is carried out by the assistant who is in charge of supporting and evaluating the students. In a Web-based experimentation setting students ask for more feedback while they work on simulation or remote manipulation. We present in this paper a virtual assistant for Web-based training. The training and the evaluation process are shared between real and virtual assistants in order to deliver a tutoring scheme adapted to Web-based experimentation.
\end{abstract}

\section{Introduction}

The Swiss Federal Institute of Technology in Lausanne (EPFL) currently supports various new learning technologies projects for promoting active and flexible learning in engineering education. The eMersion project [8] is an initiative integrated in this framework with the main objective of sustaining hands-on practice and active learning through Web-based experimentation. The Web-based experimentation environment implemented at the EPFL features Web-based simulation and remote manipulation facilities.

Web-based education is getting an increasing popularity due to its clear benefits: Classroom and platform independence. We know only four Web-based educational systems that have influenced a number of more recent systems, among which ELMART [4] and InterBook [3].

If we consider simulation, several Web-based systems exist such as Cardiac Tutor [6], Belvedere [10], and Simquest [9]. The purpose of these systems and the methods used are numerous and varied. For example, [11] use induction to generate feedback in simulation-based discovery learning. 
We propose a scheme to expand the level of support provided at EPFL [7] to students involved in Web-based experimentation activities by providing a Virtual Assistant. This solution is introduced to compensate for the students' remoteness as well as to sustain learning by providing feedback [5] or by proposing challenges to test the confidence of the student [1], [2].

During laboratory activities, a huge amount of work is traditionally performed by the assistants in charge of supporting and evaluating the students. In a Web-based experimentation framework there is a need for new cooperative learning and teaching strategies. The teaching and the evaluation process could be shared between real and virtual assistants in order to deliver an adapted teaching ${ }^{1}$.

We propose an Intelligent Tutoring System that integrates three agents: the Real Instructor, the Real Assistant (RA) and the Virtual Assistant (VA). The aim of the VA is to provide feedback during the evaluation process to reinforce learning. Since the VA is never tired, the students can interact with it at any time.

In this paper, we first describe the Cockpit-like environment the students used at EPFL to carry out Web-based experimentation. Then, we introduce a Virtual Assistant in the context of flexible learning, including some elements of justification for the VA. We also present a complete description of the goal and the functioning of the VA before going through a practical case study. We end with concluding remarks.

\section{The Cockpit-like Web-based Experimentation Environment}

\subsection{The Cockpit functionalities}

The Web-based experimentation environment provided to students has a Cockpit-like graphical user interface. This so-called Cockpit environment (Figure 1) contains all the components necessary to successfully complete laboratory assignments. In particular, the Cockpit includes two main parts: the experimentation console and the Laboratory Journal. The Cockpit also includes a navigation bar from which the students can launch useful functionalities in other browser windows, such as an experimental protocol, which describes the procedures necessary to perform the laboratory assignment.

The experimentation console can be regarded as the interactive part of the environment. It enables the actual realization of experiments. The interactions that can be sustained are mainly in the form of changes that the students can make to parameters or algorithms that effect actuations on the physical or virtual piece of equipment.

The laboratory journal is the collaborative part of the environment. It permits documenting and reporting the observations and results, and it facilitates the key activities of knowledge integration and knowledge sharing. The laboratory journal has been designed as an extended electronic version of the traditional notebook used by students to document their laboratory work.

\footnotetext{
${ }^{1}$ The term teaching here is being used to describe either the explanations given by tutors or feedback provided during the evaluation process.
} 
The laboratory journal belongs to a group (restricted access to the group members), but at any time the group can decide to make the journal visible for the teaching staff in order to get feedbacks. For this purpose, the assistant can annotate the different paragraphs of the journal. There is a journal edited for each Web-based experimentation module. A module typically corresponds to a non-consecutive 2 hours hands-on learning session.

\subsection{Initial validation of the environment}

Since October 2001, EPFL students have used a prototype of the Cockpit environment, including a basic laboratory journal, for their practical work in mechatronics. The first validation was conduced with a group of 28 volunteer students (working in pairs). They had to realize three experimental modules in mechatronics for the modeling and digital control of an electrical drive (Figure 1).

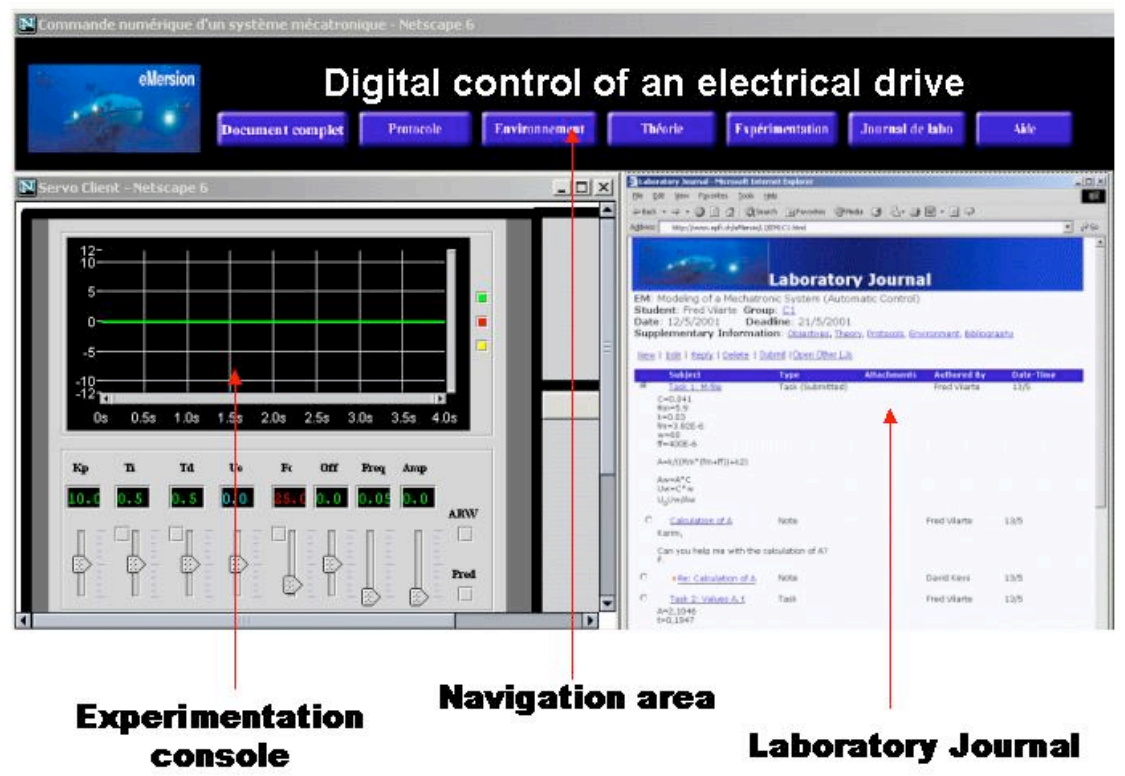

Fig. 1. Example of a Cockpit environment for Web-based experimentation in automatic control.

The progress of the experiment occurred as follow: (i) Students where asked to answer some preliminary questions listed in the protocol window (Figure 2). They answered it by editing paragraphs or attaching documents (example: script file) in their laboratory journal. When this work was finished, the students marked it visible to the assigned assistant. (ii) These questions were corrected and annotated by the assistants in the laboratory journal. (iii) Then they were authorized to perform the practical part of the experiment module using the experimentation console of the 
Cockpit. Then they were authorized to perform the practical part of the experiment module using the experimental console of the Cockpit.

1. Write a Matlab ${ }^{T M}$ script that realizes the identification of $\mathrm{A}$ and $\tau$
coefficients according to the least square method.
2. Give the numerical values of $\mathrm{A}$ and $\tau$ coefficients obtained using
your script and corresponding to the provided sample data.
3. Draw the measures and the the oretical values obtained with the
coefficients determined under number 2 .
4. Determine using an analytic method the "a priori" command to be
imposed if the reference speed $\Omega_{c}$ is 60 [rad/s].

Fig. 2. Example of preliminary questions of the protocol

The volunteers have been observed and interviewed by pedagogues to measure their reaction while they were working with such an environment. Most of them have asked for more feedback during the experimental activities and the journal editing. In the implemented flexible setting, providing the students with synchronous feedback would require for this task an assistant dedicated 24 hours a day. Thus the only way to give such feedback is to implement some automatic response features. This is done by the introduction of the VA as described in the next section. The evaluation of a laboratory journal is a very complex task strongly related to the semantic, which has nothing to do with the automatic evaluation of a quiz for example. The main consequence is that the VA can't send all the necessary feedback, but only part of it. So the VA actions are combined with the Real Assistant and Real Instructor actions.

\section{The Virtual Assistant}

\subsection{Functionalities}

In this section we introduce a Virtual Assistant (VA) using parts of an Intelligent Tutoring System (ITS). This VA is designed to give regular feedback on the structure of the laboratory journal produced by students and also on the semantics of the results and analysis. The typical role assignation is the following: (i) Structural feedback and partial semantic feedback are provided by the VA; (ii) Detailed semantic feedback is under the responsibility of the RA; (iii) Final evaluation and appreciation are handled by the Instructor.

Each laboratory journal, corresponding to an experiment, produced by a group of students goes through a life cycle, which includes interventions of the VA, the RA and the Instructor at determined steps (Figure 3). The document produced evolves from a version 1 to a version $\mathrm{N}$, until it becomes stable with respect to the evaluation and feedback given by the VA, RA and the Instructor. 


\subsection{Initialization}

When the group connects to the system for the first time, it is asked to fill a questionnaire to determine the profile of the group. Each group is assigned to a student model, which is used by the VA to customize its action. The student model is made up of two parts: (i) The cognitive part: an overlay of the capabilities of the group compared to the capabilities in the curriculum (expert knowledge); (ii) The affective part: concerning several parameters such as attention, rapidity, motivation, and confidence.

The initialization of the student model is mainly concerned by the following criteria: (i) Personal information: names, ages, addresses; (ii) Initial background: self confidence about the subject matter to be taught; (iii) Affective state: interaction, preferences for graphics, sound, video or texts, motivation, rapidity, need of support; (iv) Other parameters are not initialized until the end of the learning session, where the Instructor, the RA and the VA can provide the information.

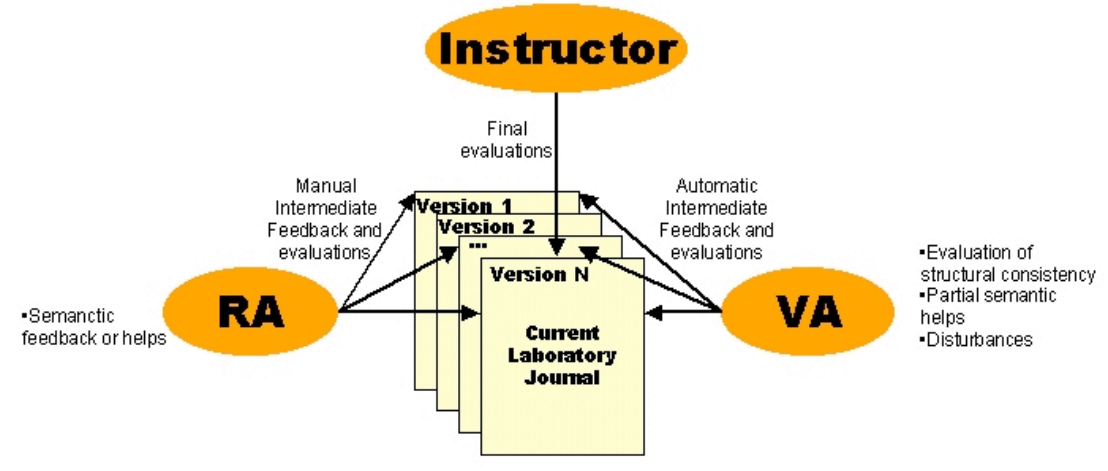

Fig. 3. The different type of feedback in the editing process of the laboratory journal.

\subsection{Structural consistency}

The laboratory journal produced by the group has to be valid with respect to a predefined structure. The aim of this structure is to ensure that students will produce organized and structured documents. It is not too restrictive for students to produce such documents because they have several degrees of freedom in editing the laboratory journal. They just have to respect some rules. To reach this goal we use XML technology, which allows high document structuring. We define a DTD (Document Type Definition) that defines the document class "laboratory journal" (Figure 4).

According to the DTD each laboratory journal is composed of a mandatory introduction, and can have 1 to $\mathrm{N}$ recursive sections. Finally a conclusion is required. Each section is composed of a mandatory title and a list of paragraphs. The paragraphs contain any type of elements: text, images formulas, links, and attached files. The student assigns a predefined type relevant to the content. The different paragraph types are: theory, configuration, measurement, observation, and analysis. 
The structural consistency is checked with two criteria analysis: (i) the consistency of the document (in XML) with the DTD of the laboratory journal. (ii) The consistency with a list of constraints.

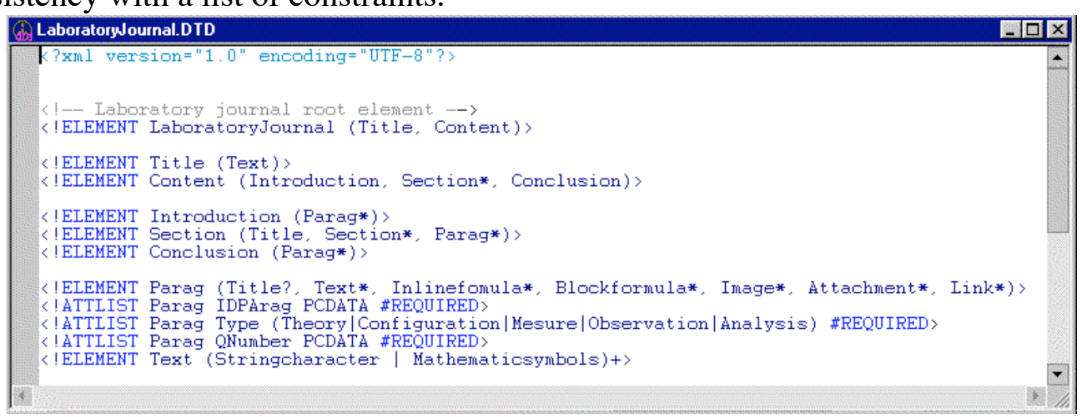

Fig. 4. DTD fragment (document Type Definition) of the laboratory journal.

We can sum up the different constraints as follow:

1. Several following paragraphs of the same type are possible,

2. A "configuration" type paragraph is followed by a paragraph of the same type or a "measurement" type,

3. A "measurement" type paragraph is followed by a paragraph of the same type or a "observation" type, or a "analysis" type,

4. A "observation" type paragraph is followed by a paragraph of the same type or a "analysis" type,

5. In a laboratory journal there is at least one instance of "configuration", "measurement" and "analysis" types.

We can see bellow (Figure 5) three sample structures of documents, answering the first two questions of the protocol given in Figure 2. They represent the same content, but the last one doesn't respect the DTD and the constraints. It has no conclusion and it doesn't respect the fourth constraint.

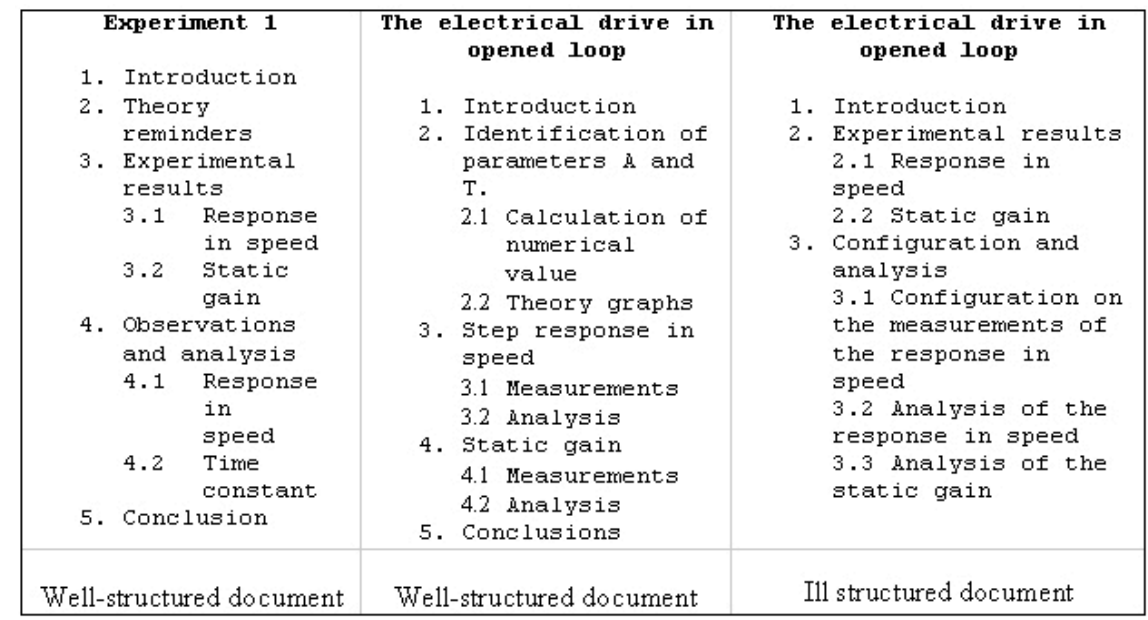

Fig. 5. Example of well-structured and ill-structured documents. 
The group submits the laboratory journal to the VA who is in charge of checking the structural consistency. By enabling structural consistency check by the VA, we ensure that the students strengthen their scientific approach and make it more rigorous.

\subsection{Semantic consistency}

There are two ways to ensure semantic consistency: an automatic one provided by the VA and a manual one by the RA (see section 4). In this section we focus on the semantic feedback given by the VA. It is called partial semantic feedback, in contrast with the feedback given by the RA, which is a more detailed semantic feedback.

There are three kinds of feedback elements given by the VA: evaluation, disturbances and hints. Disturbances are questions that are intended to test the confidence of the students about their work. Hints are documents (text, graphs, formulas, etc.) related to a particular question of the experimental protocol that help students find by themselves possible errors or problems. Disturbances and hints have been predefined and stored in a disturbance and hints databases respectively by the authors (professor, assistants). The authors may associate one or more disturbances to some learning concepts. When the VA is considering these concepts it chooses from the database the most suitable one. However, the group can ask anytime to receive a disturbance. Doing so, he can challenge or test his self-confidence with regard to the subject. When a hint is sent it is stored in a hint box that the group is free to consult or not.

Each time a group creates a new paragraph in the laboratory journal, this paragraph is identified by the paragraph type (theory, measurement, etc.) and with the question of the experimental protocol (defined by the authors in the Cockpit) it is connected with. So the VA can find which disturbance or hint in the databases correspond to it.

\subsection{Profile update}

During the work session the profile is updated with 3 different processes:

- During the working process the VA checks different actions and updates the profile with the collected information. The parameters set this way in the profile are different from the ones set by the questionnaire.

- When the work on a laboratory journal is finished there is a final evaluation by the Instructor, which is taken into account to update the profile.

- The group can update its profile at any time. Especially when it estimates it has changed its working organization, its learning strategy. Of course it cannot access the elements updated by the VA, the RA and by the Instructor. 


\section{Implementation}

\subsection{Architecture of the system}

The three different actors (VA, RA, Instructor) perform different actions such as initialization of the profile, feedback on the structure and on the semantic of the laboratory journal, profile update, and final evaluation. This tutoring scheme is represented in Figure 6 to illustrate the actors, their tasks and interactions and described with the following pseudo algorithm.

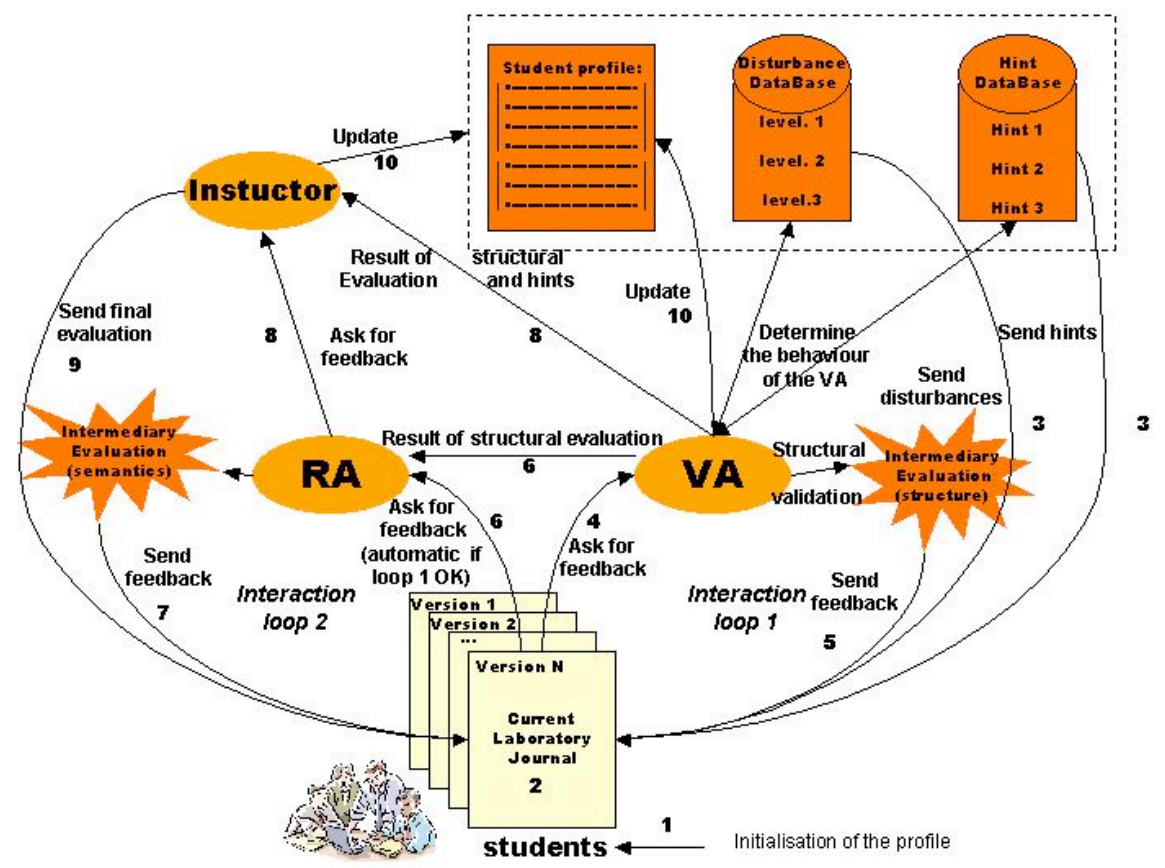

Fig. 6. Intervention of the VA, RA and Instructor in the life cycle of a laboratory journal.

1. When the group log on for the first time it has to fill a questionnaire to initialize the profile. With the result of this questionnaire the VA assign the group to a "student model".

2. Edition of the current version of the laboratory journal.

3. The VA sends some hints (partial semantic feedback) and/or disturbances (taken in the databases), for the selected paragraph, according to the student model. The consultation of the hints is optional (the hints are stored in a hint box that the group is free to consult or not). The consultation of the disturbance is required (they are automatically displayed in a pop up window). Remark: At any time the group can also ask the VA for feedback on the current paragraph.

4. The group asks the VA for a feedback on its current version of the report.

5. The VA checks the structural consistency and can also send some new hints and disturbances to the group. If the structure is correct go to step 6, else go 
back to step 2 for a new version of the journal.

6. (i) The laboratory journal is automatically sent to the RA. (ii) The VA sends the results of the structural evaluation (number of errors before a correct version, number of times where the same error is detected, number of versions, etc.) and the use of hints (number of hints used or asked), but also some other available information. Remark: The group has also the freedom to send its report and ask for feedback from the RA when it wants during steps 2 to 5.

7. The RA sends a semantic feedback (detailed semantic feedback) to the group. It also sends a general appreciation on the quality of the report. If the appreciation is correct go to step 8 else go back to step 2 .

8. The VA sends to the Instructor the final version of the report plus a summary of the evaluation.

9. The Instructor sends to the group the final evaluation (mark).

10. Information collected by the VA and the result of the final evaluation are used to update the profile.

\subsection{Example of deployment}

Let us consider as an example the completion of the prelab assignment. In an early stage, the group of students may have just answered questions 1 and 2 (Figure 2). Thus, there is still not enough material to perform a structural consistency check. However, the students are already looking for feedback at that point to make sure they have taken the right direction. Moreover, the results of question 2 are necessary to succeed to question 3 . The fact that they have been requested to write code to perform calculation on a sample data set is helpful in order to enable the virtual assistant to provide semantic consistency feedback. The virtual assistant can run the proposed code and first check whether it is free of bugs. If not, error messages can be returned with an explanatory hint. If the code is running smoothly, the concordance of the computed numerical values can by checked against the expected answers.

By having the virtual assistant handling the semantic consistency evaluation in an automatic way, it is now possible to produce different sample data set customized for each group of students. This enables to have a more personalized and efficient learning. It also helps in detecting early mistakes that may have induced further misinterpretation or motivation decrease if not detected soon enough by the students.

\section{Concluding Remarks}

The main goal of our Web-based training environment is to strengthen the knowledge acquisition. A first step has already been experimented by reintroducing hands-on experimentation in the curriculum of engineering students by means of a Web-based environment.

Students also need to benefit from a tutoring system, which is able to provide the necessary feedback while they are carrying out their experiments. A Virtual Assistant (VA) provides this feedback automatically or on request in case of:

- hints, they allow the group to realize what were its errors. In that way we support knowledge acquisition with an auto-evaluation process. 
- disturbances, they allow the group to strengthen its knowledge and its selfconfidence with regard to the domain.

- evaluations of the journal structure, they force the group to think of the best way to present its results and personal analysis.

As we can see the VA combines different types of feedback and help that have different impact in the knowledge acquisition process improving the "learning by doing approach".

The benefit of the Virtual assistant is to give more freedom and less constraints to the students seeking for feedback. In addition, the presence of a Virtual Assistant sustains the development of autonomy by being more independent of the tight protocols usually needed in a distance-learning framework.

\section{References}

1. Aïmeur, E., Frasson, C., Analysing a New Learning Strategy According to Different Knowledge Levels, Computer and Education, An International Journal, vol 27, no 2, pp. 115-127, 1996.

2. Aïmeur E., Frasson C., Dufort H., Co-operative Learning Strategies for Intelligent Tutoring Systems, Applied Artificial Intelligence. An International Journal, Vol 14(5), pp. 465-490, 2000.

3. Brusilovsky P., Eklund J., Schwartz E., Web-based education for all: A tool for developing adaptive courseware. Computer Networks and ISDN Systems. 30, 1-7, pp. 291-300, 1998.

4. Brusilovsky P., Schwartz E., Weber G., ELM-ART: An Intelligent tutoring system on World Wide Web. ITS'96 Conference, Third International Conference on Intelligent Tutoring Systems. Lecture Notes in Computer Sciences, no 1086, Frasson C., Gauthier G., Lesgold A. Editors, Springer Verlag, Montréal, pp. 261-269, 1996.

5. Callear D., Intelligent tutoring environment as teacher substitutes: Use and feasibility, Educational technology journal, September - October 1999, pp.6-8, 1999

6. Eliot C.R., Woolf B., Reasoning about the user within a simulation-based-real time training system, Fourth International Conference on User Modeling, Hyannis Cape Cod, Mass, pp. 15-19 August, 1994.

7. Gillet D., Salzmann C., Latchman H.A., and Crisalle O.D., Advances in Remote Experimentation, 19th American Control Conference, Chicago, Illinois, USA, pp. 29552956, 2000

8. Gillet D., Fakas G., eMersion: A new paradigm for Web-based training in engineering education, International Conference on Engineering Education, pp. 8B4-10 - 8B4-14, Oslo, 2001.

9. Jong T., Joolingen W.T., King S., The authoring Environment SimQuest and the need for author support. Supporting authors in the design of simulation-based-discovery environment. Servive project deliverable D 8.1. Enschede, University of Twente, 1997.

10. Suthers D., and Jones D., An architecture for Intelligent Collaborative Educational Systems, AI-Ed 97, World Conference on Artificial Intelligence and Education, Japan, pp. 55-62, 1997.

11. Veermans K., and Van Joolingen W.R., Using Induction to generate Feedback in Simulation Based Discovery Learning Environment, ITS-98, Fourth International Conference on Intelligent Tutoring Systems, Lecture Notes in Computer Sciences, no. 1452, Goettl B.P., Halff H.M., Redfield C.L., Shute V.J. Editors, Springer Verlag, San Antonio, Texas, pp. 196-205, 1998. 